\title{
Combinations of the spherical harmonics and the spherical rotator functions invariant with respect to the symmetry group $O_{h}$
}

\author{
K. W. Wojciechowski \\ Institute of Molecular Physics, Polish Academy of Sciences \\ Smoluchowskiego 17/19, 60-179 Poznań, Poland \\ kww@man.poznan.pl
}

Abstract: The coefficients sufficient to construct all the invariant (with respect to elements of the symmetry group $O_{h}$ ) cubic harmonics and the invariant cubic rotator (known also as the Wigner) functions are given up to $l=30$.

Description of various problems in which symmetry plays an important role can be much simplified if one uses functions which are either invariant under action of elements of the symmetry group or which transform in a convenient way under such actions. Interaction of molecules of axial symmetry can be described by the spherical harmonics, $Y_{l m}(\theta, \phi)$, where the angles $(\theta, \phi)$ define the orientation of the symmetry axis of a molecule [1], If the molecules are not axially symmetric, the spherical rotator functions, $D_{m n}^{l}(\Omega)$, are necessary, in general; $\Omega$ defines the orientation of the molecule and, usually, is expressed by the Euler angles $(\phi, \theta, \chi)$. Spherical harmonics in a primed system, obtained from an unprimed system by a rotation $\hat{\Omega}$, can be expressed by the spherical harmonics in the unprimed system:

$$
\hat{\Omega} Y_{l m}(\theta, \phi)=Y_{l m}\left(\theta^{\prime}, \phi^{\prime}\right)=\sum_{l=-m}^{m} Y_{l m}(\theta, \phi) D_{k m}^{l}(\Omega) .
$$

For non-axial molecules, of the symmetry group $G$, it is convenient to use certain combinations of the spherical harmonics or the spherical rotator functions which are adapted to this symmetry, i.e. are transformed in a convenient manner under action of elements of the symmetry group of the molecules [2], We will denote these symmetry adapted harmonics and symmetry adapted rotator functions by $Y_{l m}^{(G)}(\theta, \phi)$ and $D_{k m}^{(G) l}(\Omega)$, respectively. These functions fulfill the relations analogous to the Eq.(1): 


\begin{tabular}{|c|c|c|c|c|c|c|c|c|}
\hline$l_{\mu}$ & $A_{\mu, 0}^{l}$ & $A_{\mu, \pm 4}^{l}$ & $A_{\mu, \pm 8}^{l}$ & $A_{\mu, \pm 12}^{l}$ & $A_{\mu, \pm 16}^{l}$ & $A_{\mu, \pm 20}^{l}$ & $A_{\mu, \pm 24}^{l}$ & $A_{\mu, \pm 28}^{l}$ \\
\hline 4 & $\sqrt{\frac{7}{12}}$ & $\sqrt{\frac{5}{24}}$ & & & & & & \\
\hline 6 & $\frac{\sqrt{2}}{4}$ & $-\frac{\sqrt{7}}{4}$ & & & & & & \\
\hline 8 & $\frac{\sqrt{33}}{8}$ & $\sqrt{\frac{7}{96}}$ & $\sqrt{\frac{65}{384}}$ & & & & & \\
\hline 10 & $\sqrt{\frac{65}{384}}$ & $-\frac{\sqrt{11}}{8}$ & $-\sqrt{\frac{187}{768}}$ & & & & & \\
\hline $12_{1}$ & $\sqrt{\frac{743}{1536}}$ & $5 \sqrt{\frac{3003}{1521664}}$ & $\frac{1}{32} \sqrt{\frac{46189}{743}}$ & $\sqrt{\frac{676039}{4564992}}$ & & & & \\
\hline $12_{2}$ & & $\frac{1}{8} \sqrt{\frac{7429}{743}}$ & $-\sqrt{\frac{483}{1486}}$ & $\frac{9}{8} \sqrt{\frac{11}{743}}$ & & & & \\
\hline 14 & $\frac{1}{32} \sqrt{\frac{595}{3}}$ & $\frac{-1}{64} \sqrt{429}$ & $-\sqrt{\frac{247}{2048}}$ & $\frac{-1}{64} \sqrt{\frac{2185}{3}}$ & & & & \\
\hline $16_{1}$ & $\frac{1}{128} \sqrt{\frac{22819}{3}}$ & $\frac{11}{64} \sqrt{\frac{1547}{1201}}$ & $7 \sqrt{\frac{25445}{29515776}}$ & $\frac{5}{64} \sqrt{\frac{10557}{1201}}$ & $\sqrt{\frac{5272635}{39354368}}$ & & & \\
\hline $16_{2}$ & & $5 \sqrt{\frac{4991}{614912}}$ & $\frac{-1}{8} \sqrt{\frac{4185}{1201}}$ & $-\sqrt{\frac{387283}{1844736}}$ & $\frac{1}{4} \sqrt{\frac{1885}{3603}}$ & & & \\
\hline $18_{1}$ & $\sqrt{\frac{6871}{32768}}$ & $-13 \sqrt{\frac{24871}{56287232}}$ & $\frac{-35}{128} \sqrt{\frac{7429}{6871}}$ & $-5 \sqrt{\frac{215441}{56287232}}$ & $\frac{-1}{256} \sqrt{\frac{648223995}{6871}}$ & & & \\
\hline $18_{2}$ & & $5 \sqrt{\frac{4669}{10553856}}$ & $\frac{-1}{8} \sqrt{\frac{268279}{20613}}$ & $167 \sqrt{\frac{33}{3517952}}$ & $\frac{-1}{4} \sqrt{\frac{2635}{6871}}$ & & & \\
\hline $20_{1}$ & $\sqrt{\frac{59087}{131072}}$ & $\frac{13}{512} \sqrt{\frac{17765}{367}}$ & $\frac{15}{265} \sqrt{\frac{3553}{367}}$ & $5 \sqrt{\frac{290377}{192413696}}$ & $\frac{5}{512} \sqrt{\frac{187891}{367}}$ & $\sqrt{\frac{23783045}{192413696}}$ & & \\
\hline $2 \mathrm{O}_{2}$ & & $\frac{7}{64} \sqrt{\frac{13485}{734}}$ & $\frac{-1}{8} \sqrt{\frac{899}{2202}}$ & $\frac{-129}{128} \sqrt{\frac{33}{367}}$ & $\frac{-17}{4} \sqrt{\frac{17}{2202}}$ & $\frac{1}{128} \sqrt{\frac{776815}{1101}}$ & & \\
\hline $22_{1}$ & $\frac{1}{512} \sqrt{\frac{173965}{3}}$ & $-85 \sqrt{\frac{39767}{4974968832}}$ & $-3 \sqrt{\frac{2800733}{414580736}}$ & $\frac{-9}{1024} \sqrt{\frac{2750041}{3163}}$ & $-5 \sqrt{\frac{53553343}{1658322944}}$ & $\frac{-1}{1024} \sqrt{\frac{407771117}{3163}}$ & & \\
\hline $22_{2}$ & & $\frac{3}{64} \sqrt{\frac{33263}{3163}}$ & $\frac{-1}{8} \sqrt{\frac{136493}{9489}}$ & $43 \sqrt{\frac{481}{77733888}}$ & $\frac{11}{4} \sqrt{\frac{247}{9489}}$ & $-25 \sqrt{\frac{5453}{77733888}}$ & & \\
\hline
\end{tabular}




\begin{tabular}{|c|c|c|c|c|c|c|c|c|}
\hline $24_{1}$ & $\frac{1}{1024} \sqrt{\frac{924397}{2}}$ & $\frac{1615}{1024} \sqrt{\frac{9867}{924397}}$ & $\frac{15}{2048} \sqrt{\frac{955049953}{1848794}}$ & $\frac{385}{1024} \sqrt{\frac{392863}{1848794}}$ & $\frac{35}{2048} \sqrt{\frac{109402007}{924397}}$ & $\frac{1}{1024} \sqrt{\frac{87670790155}{1848794}}$ & $\frac{5}{2048} \sqrt{\frac{35830670759}{1848794}}$ & \\
\hline $24_{2}$ & & $\frac{\sqrt{12671037068063505}}{236645632}$ & $\frac{-167219}{384} \sqrt{\frac{323}{9396393821330}}$ & $\frac{-7823363}{768} \sqrt{\frac{2717}{9396393821330}}$ & $\frac{-10805143}{96} \sqrt{\frac{37}{4698196910665}}$ & $\frac{-379415}{768} \sqrt{\frac{717541}{1879278764266}}$ & $\frac{9523}{384} \sqrt{\frac{775661821}{9396393821330}}$ & \\
\hline $24_{3}$ & & & $\frac{7}{96} \sqrt{\frac{33724427}{5082445}}$ & $\frac{-1}{24} \sqrt{\frac{677554397}{5082445}}$ & $\frac{1}{12} \sqrt{\frac{294405133}{10164890}}$ & $\frac{-35}{24} \sqrt{\frac{15181}{1016489}}$ & $\frac{49}{96} \sqrt{\frac{7429}{5082445}}$ & \\
\hline $26_{1}$ & $\frac{1}{2048} \sqrt{964743}$ & $\frac{-323}{1024} \sqrt{\frac{805}{1706}}$ & $\frac{-55}{4096} \sqrt{\frac{230299}{853}}$ & $\frac{-55}{2048} \sqrt{\frac{184667}{2559}}$ & $\frac{-1}{2048} \sqrt{\frac{416424085}{1706}}$ & $\frac{-5}{2048} \sqrt{\frac{10110805}{853}}$ & $\frac{-7}{4096} \sqrt{\frac{95041567}{2559}}$ & \\
\hline $26_{2}$ & & $\frac{11}{256} \sqrt{\frac{47027}{2559}}$ & $\frac{-1}{128} \sqrt{\frac{17149685}{5118}}$ & $\frac{-61}{\frac{256}{\frac{205}{1706}}}$ & $\frac{127}{32} \sqrt{\frac{11}{2559}}$ & $\frac{79}{256 \sqrt{\frac{6923}{5118}}}$ & $\frac{-1}{128} \sqrt{\frac{1626905}{1706}}$ & \\
\hline $28_{1}$ & $\sqrt{\frac{1}{4096} \sqrt{\frac{21791791}{3}}}$ & $\frac{7429}{8192} \sqrt{\frac{5655}{200846}}$ & $\frac{1045}{8192} \sqrt{\frac{147407}{100423}}$ & $\frac{2145}{8192} \sqrt{\frac{74037}{200846}}$ & $\frac{1}{4096} \sqrt{\frac{93326970165}{200846}}$ & $\frac{5}{8192} \sqrt{\frac{17337421335}{200846}}$ & $\frac{3}{8192} \sqrt{\frac{31740817521}{100423}}$ & $\frac{1}{\frac{1}{8192} \sqrt{\frac{4405927765415}{602538}}}$ \\
\hline $28_{2}$ & & $\frac{11}{2048} \sqrt{\frac{795453861}{100423}}$ & $\frac{3173}{768} \sqrt{\frac{3110405}{33472391822}}$ & $\frac{-480517}{6144} \sqrt{\frac{64285}{50208587733}}$ & $\frac{-93719}{192} \sqrt{\frac{10373}{50208587733}}$ & $\frac{-9606403}{6144} \sqrt{\frac{1927}{50208587733}}$ & $\frac{-15757}{768} \sqrt{\frac{14905345}{100417175466}}$ & $\frac{6925}{\frac{6948}{204828280461}}$ \\
\hline $28_{3}$ & & & $\frac{1}{192 \sqrt{\frac{438417551}{166657}}}$ & $\frac{-11}{96} \sqrt{\frac{154950007}{999942}}$ & $\frac{-1}{24} \sqrt{\frac{51935}{999942}}$ & $\frac{73}{96} \sqrt{\frac{279565}{999942}}$ & $\frac{-805}{192} \sqrt{\frac{1771}{499971}}$ & $\frac{7}{16} \sqrt{\frac{6095}{999942}}$ \\
\hline $30_{1}$ & $\frac{1}{4096} \sqrt{\frac{23859587}{6}}$ & $\frac{-6555}{16384} \sqrt{\frac{5883955}{23859587}}$ & $\frac{-35}{8192} \sqrt{\frac{106010544783}{47719174}}$ & $\frac{-715}{16384} \sqrt{\frac{5332239153}{23859587}}$ & $\frac{-195}{8192} \sqrt{\frac{1935208077}{23859587}}$ & $\frac{-21}{16384} \sqrt{\frac{752444085939}{23859587}}$ & $\frac{-7}{8192} \sqrt{\frac{12416705520715}{143157522}}$ & $\frac{-1}{16384} \sqrt{\frac{626401385407105}{23859587}}$ \\
\hline $30_{2}$ & & $\frac{1}{1024} \sqrt{\frac{1081849628587}{23859587}}$ & $\frac{-3023519}{64} \sqrt{\frac{89585}{1121899874717562}}$ & $\frac{-18820217}{1024} \sqrt{\frac{16445}{186983312452927}}$ & $\frac{171091}{16} \sqrt{\frac{47515}{560949937358781}}$ & $\frac{1998239}{1024} \sqrt{\frac{13227445}{560949937358781}}$ & $\frac{51991}{64} \sqrt{\frac{45951477}{373966624905854}}$ & $\frac{-49921}{1024} \sqrt{\frac{5370752933}{186983312452927}}$ \\
\hline $30_{3}$ & & & $\frac{1}{\frac{1}{128} \sqrt{\frac{628003519}{15673642}}}$ & $\frac{-37}{128} \sqrt{\frac{6105441}{7836821}}$ & $\frac{\frac{3}{32} \sqrt{\frac{203562029}{7836821}}}{4}$ & $\frac{-25}{128} \sqrt{\frac{35830067}{7836821}}$ & $\frac{1851}{\frac{128}{\frac{2185}{15673642}}}$ & $\frac{-5}{\frac{-5}{64} \sqrt{\frac{770385}{7836821}}}$ \\
\hline
\end{tabular}

Table 1. The coefficients $A_{\mu k}^{l} \equiv a_{\mu k}^{\left(O_{h}\right) l}$ are sufficient to construct, according to Eqs. (5) and (6), all the invariant cubic harmonics and the invariant cubic rotator functions up to $l=30$. Empty spaces in the table above correspond to coefficients equal zero. All the coefficients not shown in the table are also equal zero. 


$$
\hat{\Omega} Y_{l m}^{(G)}(\theta, \phi)=Y_{l m}^{(G)}\left(\theta, \phi^{\prime}\right)=\sum_{l=-m}^{m} Y_{l m}^{(G)}(\theta, \phi) D_{k m}^{(G) l}(\Omega)
$$

The harmonics $Y_{l m}^{(G)}(\theta, \phi)$ form a complete orthonormal set on the 3D unit sphere:

$$
\int_{0}^{2 \pi} d \phi \int_{0}^{\pi} d \theta \sin \theta\left[Y_{l m}^{(G)}(\theta, \phi)\right]^{*} Y_{l^{\prime} m^{\prime}}^{(G)}(\theta, \phi)=\delta_{l^{\prime} l} \delta_{m^{\prime} m},
$$

whereas the rotator functions $D_{k m}^{(G) l}(\Omega)$ form a complete orthogonal set (they are normalized to $\left.8 \pi^{2} /(2 l+1)\right)$ on the $4 \mathrm{D}$ unit sphere:

$$
\int_{0}^{2 \pi} d \phi \int_{0}^{\pi} d \theta \sin \theta \int_{0}^{2 \pi} d \chi\left[D_{m^{\prime} n^{\prime}}^{(G) l^{\prime}}(\phi, \theta, \chi)\right]^{*} D_{m n}^{(G) l}(\phi, \theta, \chi)=\frac{8 \pi^{2}}{2 l+1} \delta_{l^{\prime} l} \delta_{m^{\prime} m} \delta_{n^{\prime} n} .
$$

The symmetry adapted functions which are invariant with respect to all elements of the symmetry group $G$ are of special interest. If the symmetry adapted harmonics of such a property are written as:

$$
Y_{l \mu}^{(G)}(\theta, \phi)=\sum_{l=-m}^{m} a_{\mu k}^{(G) l} Y_{l k}(\theta, \phi)
$$

then the invariant (fully symmetric with respect to the elements of the group $G$ ) symmetry adapted rotator functions have the form:

$$
D_{\mu \nu}^{(G) l}(\theta, \phi)=\sum_{k=-m}^{m} \sum_{k^{\prime}=-m}^{m}\left[a_{\mu k}^{(G) l}\right]^{*} D_{k k^{\prime}}^{l}(\theta, \phi) a_{v k}^{(G) l} .
$$

The following example concerns the case when $G=O_{h}$, i.e. the case of the cubic group with the inversion centre.

Low order cubic harmonics $(l \leq 8)$ were obtained already by Von der Lage and Bethe [3]. The cubic harmonics up to $l=12$ were later calculated in [4] by the projection operator technique. At present, the invariant cubic harmonics (transforming according to the fully symmetric representation of the cubic point group, $O_{h}$ ) of even higher order can be obtained. Such calculations, which are practically impossible to perform "by hand" for $l>12$, can be done successfully using symbolic programming; here we used Mathematica [5], In Table 1 we present the expansion coefficients of all the invariant cubic harmonics into the spherical harmonics up to $l=30$. These coefficients can be useful for calculations concerning systems of the $O_{h}$ symmetry group when high accuracy is required. 


\section{Acknowledgments}

This work was supported by the Committee for Scientific Research (KBN) within the grant $8 \mathrm{~T} 11 \mathrm{~F} 01008 \mathrm{p} 04$.

\section{References}

[1] C. G. Gray and K. E. Gubbins, Theory of Molecular Fluids, Clarendon Press, Oxford, 1984.

[2] H. M. James and T. A. Keenan, J. Chem. Phys. 31, 12 (1959).

[3] F. C. Von der Lage and H. A. Bethe, Phys. Rev. 71, 612 (1947).

[4] S. L. Altmann and A. P. Cracknell, Rev. Mod. Phys. 37, 19 (1965).

[5] S. Wolfram, Mathematica, A System for Doing Mathematics by Computer, Wolfram Res. Inc. (1992). 